Le genre de la maladie : pratiques, discours, textes et représentations

\title{
La rage contre le Moi, l'Autre et Dieu dans la trilogie de Linda Lê: une thérapeutique
}

Thu Thuy BUI

\section{(2) OpenEdition Journals}

Electronic version

URL: http://journals.openedition.org/transtexts/663

DOI: $10.4000 /$ transtexts. 663

ISSN: 2105-2549

Publisher

Gregory B. Lee

Electronic reference

Thu Thuy BUI, «La rage contre le Moi, l'Autre et Dieu dans la trilogie de Linda Lê: une thérapeutique », Transtext(e)s Transcultures 跨文本跨文化 [Online], 11 | 2016, Online since 30 June 2017, connection on 19 April 2019. URL : http://journals.openedition.org/transtexts/663 ; DOI : 10.4000/transtexts.663

This text was automatically generated on 19 April 2019

(c) Tous droits réservés 


\section{La rage contre le Moi, l'Autre et Dieu dans la trilogie de Linda Lê: une thérapeutique}

Thu Thuy BUI

1 En revendiquant d'être une métèque écrivant en français, Linda Lê, née au Vietnam en 1963, fait entendre une plainte et une souffrance affirmant sa non-appartenance. Avant son arrivée en France à quatorze ans, elle fréquentait déjà l'école française. Écrivant un français exigeant et recherché dans ses effets baroques, mélangeant mots rares, savants, argotiques et expressions populaires, Linda Lê est considérée comme une auteure difficile dont les œuvres constituent un métissage souvent sous-jacent d'aspects littéraires et culturels de l'Orient et de l'Occident. À travers ses œuvres, elle construit un univers sombre où l'expérience de l'exil est profondément marquée par la perte, le deuil, le double, la folie, la mort.

2 Cet article se focalise sur la trilogie consacrée à la mort du père et il est structuré en trois parties qui caractérisent la rage destructrice à travers d'abord «Les mots rageurs et les idées mortifères », ensuite « Les images et les symboles obsessionnels » et enfin « La rage : autres je(ux) avec le feu ». Son objectif est de montrer que le recours à l'écriture a permis à l'écrivain en crise de retrouver sa voie/voix et que ses mots écrits sont une thérapie face aux maux vécus.

3 La mort de son père, sans que l'écrivaine ne l'ait jamais revu, l'a conduite au bord de la folie. C'est dans ce contexte qu'elle écrit la trilogie : Les Trois Parques (1997), Voix : une crise (1998), Lettre morte (1999). À ce sujet, Linda Lê confie :

Lorsque mon père est mort au Vietnam, en 1995, j'ai vécu pendant deux ans
enceinte d'une très très grande douleur qui n'a vu le jour qu'avec
l'achèvement des Trois Parques. Je souffrais d'hallucinations, de pensées
suicidaires, de conduites paranoïaques. Tant que mon père était en vie, tous
mes livres lui étaient adressés. Il était mon lecteur idéal, mon lecteur
imaginaire. Lorsqu'il est mort, j'ai perdu mon lecteur, le ciel est devenu
désert, le monde sans Dieu. Tout était sans voix, il n'y avait plus d'écho à ce
que je pouvais écrire. J'ai écrit Les Trois Parques dans un état de solitude 
absolue et volontaire. Et c'est pour cela qu'il y a dans ce livre un véritable déchaînement verbal, parce que j'étais cloîtrée en moi-même. ${ }^{1}$

4 La trilogie se déroule autour d'un père abandonné par les siens, resté seul au pays où il meurt dans la solitude. Dans le premier volet, mort et mots s'opposent et l'écrivaine endeuillée crache le venin de sa rage. C'est un déferlement verbal qui déverse sans frein les tumultes de la pensée comme le lâcher d'eau d'un barrage. L'auteure use de toute la puissance de la fiction et nous fait sentir l'impossible deuil après la mort de son père. Le récit commence dans la cuisine avec la discussion sur le projet d'inviter en France le vieux père surnommé « roi Lear » et il est interrompu par différentes couches de récits sur la vie de chaque personnage, les souvenirs d'enfance au Vietnam, la situation sociopolitique vietnamienne d'autrefois et d'aujourd'hui et enfin se termine par l'annonce de la mort du père qui rend impossible le projet initial. Dans le deuxième volet, mort et mal se mêlent. On peut distinguer deux parties : la première évoque ce qui se passe dans un "centre de crise " où se trouve la narratrice et la deuxième décrit ses hallucinations auditives et visuelles. Avec le troisième volet, la mort et l'amour se retrouvent dans la même tombe. Il s'agit d'un long monologue - 96 pages d'un seul paragraphe - qui résonne comme un poème en prose, d'un seul bloc. Ce monologue intérieur donne directement accès à la conscience de la narratrice-héroïne, qui enchaîne une idée après l'autre sous les couleurs de la culpabilité, de la nostalgie et de l'amertume.

Cette trilogie se construit sur un conflit intérieur entre passé et présent, ici et là-bas, amour et haine. L'écrivaine y déclenche sa révolte contre le Moi, le Monde, Dieu, contre ce qu'Emmanuel Kant appelle les trois idées transcendantales. ${ }^{2}$

\section{Les mots rageurs et les idées mortifères}

6 L'écriture et la rage sont en effet étroitement solidaires dans la trilogie. Une conscience aiguë du double (double culture, double identité...) se manifeste à travers les textes nocifs et extrémistes d'une écrivaine qui a «toujours été fascinée par des figures de reclus, de renoncement, des figures qui exigent toujours plus de soi ».3 Écrire sur le double revient à s'installer sur une ligne de crête sans cesse menacée et menaçante entre le Moi et l'Autre.

Dans Les Trois Parques, le père vivant est évoqué tout au long du livre et sa mort n'intervient qu'à la fin à travers une vision mythique (il s'envole vers le ciel). Le livre est dominé par un « déchaînement » tant dans les thèmes que dans les mots. Linda Lê dit à ce propos: "l'anathème, l'imprécation [...] sont contenus dans Les Trois Parques, c'est une façon de prendre Dieu pour cible $»^{4}$ ou encore: "Tout a jailli comme d'un volcan en éruption, le chagrin s'était transformé en rage $\$ .{ }^{5}$ L'écrivaine met en situation trois protagonistes féminins. On ne connaît que leur surnom: "Ventre rond", l'ainée des sœurs, "Belles Gambettes", la cadette et la cousine "la Manchote». Néanmoins, la Manchote qui se sent coupée de la normalité, incarne une part de Linda Lê, comme elle l'avoue: «Dans Les Trois Parques, si je me reconnais un tant soit peu dans un personnage, c'est celui de la Manchote, pythie qui prêche dans le désert, qui refuse d'appartenir à une communauté ». ${ }^{6}$ Physiquement, il manque à la Manchote une main alors que les deux autres montrent au contraire un corps épanoui (l'une est enceinte, l'autre a de longues jambes) ; mentalement, la Manchote n'a « rien d'autre à faire qu'à se torturer la cervelle, essayer de se rendre un peu plus malheureuse $»^{7}$ et quand il y a des réunions familiales, elle reste dans son coin pour observer et critiquer. N'étant jamais contente des autres ni 
d'elle-même, elle critique tout. Elle se sent «de nulle part » et en souffre. La lassitude qu'elle éprouve d'elle-même et le désespoir qui en découle éveillent en elle une tentation suicidaire.

Le thème de la nourriture, très présent dans Les Trois Parques, constitue le seul lien qui relie vraiment les personnages les uns aux autres: les scènes où l'aînée reprend les recettes de sa grand-mère vietnamienne répondent à celles où le père prépare la cuisine au Vietnam. Et même l'exception qu'offre la Manchote confirme la règle. Dépourvue de toute gourmandise, quasiment au bord de l'anorexie, elle crache sur tous les plats préparés selon les recettes de la grand-mère, et retient à table son souffle pour ne pas être intoxiquée. On évoque même le souvenir du repas de mariage de Ventre rond, où la Manchote reste à l'écart, immobile à table, ne mangeant ni ne parlant. Son comportement alimentaire est comme son comportement social: marginal et troublant. Plus elle se claustre en soi, moins elle a d'élan vers l'autre.

9 Le personnage du père, contrairement à ce qu'on pourrait supposer, est décrit sur un ton sarcastique. L'auteure ne manque pas de mots péjoratifs pour l'identifier : «vieil idiot», « filou », « vieux brigand », « vieil escobar », " père périmé », « vieille bête de père »... Elle ne lui montre aucun respect :

Vingt ans que le roi Lear regardait le monde tourner sans lui. Vingt ans qu'il n'avait pas bougé de la maison bleue, où mes cousines l'avaient laissé [...] Et maintenant, il voyait le retour des saprophytes. Le roi Lear ricanait, assis derrière sa fenêtre. Le palais en ruine, au milieu des nouvelles villas blanches, se tassait de plus en plus sur lui-même, comme un vieillard dans un lit de vierges. ${ }^{8}$

Pourquoi l'auteure présente-elle le père sous un jour aussi piteux ? Est-ce une attitude de « contre-tout » pendant la première étape du deuil ? Est-il possible qu'en écrivant ce livre, elle soit guidée par le raisonnement « puisque que le père m'a abandonnée, je lui en veux » ou " puisqu'il me fait mal, je me venge »? C'est possible. D'ailleurs, sa vengeance retombe sur tous les personnages des Trois Parques, dépeints de façon négative : la grandmère, le curé, Théo... Théo, compagnon de Belles Gambettes, est le seul Français et le seul qui porte un nom. (Théo, Dieu en grec, est décrit comme : vendeur d'orviétan, jongleur de rêves, trafiquant de mirages, parasite...) La figure de Dieu est intimement liée à celle du père à travers le personnage du curé, son seul ami. L'auteure le dépeint sur un mode ironique: gourmand, amateur d'anguilles, ayant perdu la foi et se sentant perpétuellement frustré. Dieu est également affublé de surnoms dévalorisants : Dépeceur, Lâcheur, Grand Sourd, Chevillard. D'une part, «le Grand Sourd ne bronchait pas, caché derrière le paravent bleu, à épier le spectacle de ses marionnettes ", d'autre part, le curé ne croit plus en Dieu : il «ne priait plus, ne demandait plus rien au Chevillard d'en haut [...] ne disait plus la messe $" .^{9}$ Devenu fou, il met le feu à son église avant de disparaître dans son habit noir couvert de cendres. La fin du roman exprime une rupture définitive avec Dieu. Paradoxalement, c'est le prêtre, considéré comme fils de Dieu le Père, qui a déclenché l'incendie. L'enfant a trahi son Père, comme, le "roi Lear » est trahi par ses filles. Freud a écrit que «le dieu de chacun est l'image de son père, que l'attitude personnelle de chacun à l'égard du dieu dépend de son attitude à l'égard de son père charnel $»^{10}$ De là à ce que la mort du père entraîne celle de Dieu...

Dans Voix : une crise, récit halluciné, Dieu fait de nouveau l'objet d'imprécations : «Si Dieu me rend visite, je Lui cracherai à la gueule », «Dieu le meilleur ennemi de l'homme ", 
«Dieu est mort », «Le Christ est entré au FBI », «Dieu a empoisonné l'air, s'll me rend visite cette nuit, je Lui sauterai à la gorge $» \cdot{ }^{11}$ La narratrice qui vient de perdre son père est sous l'emprise des voix : celle du passé et celle du présent, celle qui est dans le réel et celle qui est dans le rêve... Elle recourt donc à l'Autre pour vaincre son incertitude existentielle, car c'est seulement à travers la prise en compte de l'Autre que le Moi peut se situer. Mais l'Autre constitue aussi une menace. En l'occurrence, l'Autre est «l'Organisation », métaphore du monde auquel la narratrice n'appartient plus. Dans son délire, elle voit les envoyés de l'organisation qui guettent ses gestes, qui font des gammes sur ses nerfs. Il en naît une obsession du suicide, qui peut être paradoxalement interprétée comme une sorte de défense : se tuer avant que l'Autre ne la tue. Pour lutter contre la menace de mort, la narratrice se précipite dans l'autodestruction : « De ma main vide, je serre fort mon cou, j'enfonce les ongles dans la chair. Je me frappe la tête contre le mur", "Sommeil agité par des rêves où j'étais poursuivie par la meute de l'Organisation [...] Je me jette à l'eau pour échapper à la meute $» .^{12}$ Sa confusion mentale est telle qu'elle s'imagine être poursuivie par l'Organisation jusque dans son pays natal: "J'erre dans les rues du pays de mon enfance. La ville est déserte. Partout ruines et cendres [...] L'Organisation a saccagé la ville, pour que je ne puisse trouver refuge nulle part $» .^{13}$ Terrifiée par l'angoisse d'être prise au piège, elle voit partout les membres de l'Organisation :

L'Organisation tient toute la ville, tout le pays. Ceux que j'appelle au secours rejoignent aussitôt le Grand Complot, se moquent de moi, me scrutent, menacent de m'envoyer chez ces Grands Inquisiteurs, les psychiatres [...] Ils ne cherchent qu'à m'empoisonner, à m'endormir [...] Ils veulent que je me sacrifie, ils tentent d'étouffer ma voix, de bâillonner mes cris. ${ }^{14}$

Pour le troisième volet, Lettre morte, écrit sous forme d'un monologue, l'Autre est l'amant que la narratrice endeuillée nomme "Morgue ». Plus la culpabilité vis-à-vis du père est grande, plus la haine contre Morgue augmente. Les accusations qu'elle porte contre son amant sont autant d'autojustifications. En effet, il est la cause du délaissement et de la négligence dont la narratrice fait preuve envers son père. L'auteure fait coïncider la mort $\mathrm{du}$ père et la fin d'une relation amoureuse passionnelle, ce qui amplifie la rage. Culpabilité, souffrance, folie atteignent un niveau paroxystique: "Je ressemble à une loque, à un oiseau blessé qui traîne ses ailes. Je porte un mort sur mes épaules", "J'attendais qu'on vînt me dépouiller, m'assassiner à coups de couteau, me délivrer de ce corps, corps infirme, corps vendu, corps souillé, qui soulevait des vagues de dégoût en moi », «Je portais ma souffrance comme une femme porte une orpheline pour laquelle elle ne cherche rien d'autre qu'un tombeau ", "Je n'aurais plus eu d'autre pensée que celle de disparaitre à jamais, noyée dans la solitude et la déraison $» .{ }^{15}$ D'une part, la narratrice déclare haïr son corps et se dégoûter d'elle-même. D'autre part, elle éprouve un sentiment apocalyptique et dysphorique envers l'Autre à travers son désir de solitude.

Ainsi, l'écrivaine nous amène aux différents états sentimentaux, mélange de reproches amers faits à elle-même et d'accusations agressives contre l'Autre. C'est par le rythme haletant de l'écriture, par le ton hargneux, caustique, sarcastique, par les idées noires que l'auteure choisit de montrer sa rage. 


\section{Les images et les symboles obsessionnels}

14 Il est intéressant de mettre en rapport certaines des images et des symboles, qui regorgent de pessimisme et de souffrance, parce que Linda Lê y a subtilement transcrit ses angoisses et ses imaginaires extravagants.

Le feu est un symbole particulièrement fort chez Linda Lê. C'est avec le feu que le curé dans Les Trois Parques détruit l'église et que la fille endeuillée dans Voix et Lettre morte élimine les lettres du père. Mais le feu se présente aussi sous une forme ondoyante et fluide. L'image d'un père qui se brûle la langue avec l'alcool - «l'eau de feu » pour reprendre le terme de Bachelard - traverse toute l'œuvre de Linda Lê. Il est notamment lié à l'image du père mort : « Mon père m'est apparu cette nuit. Il était enveloppé dans un manteau de feu. Il me demandait pourquoi je l'avais tué une seconde fois en brûlant les lettres ", "Mon père apparaît à ma fenêtre, une boule de feu entre ses mains ", "Mon père est allongé sur une table de dissection. Autour de lui, les chiens de l'enfer veillent. Leurs gueules crachent du feu », «Mon père est debout sur une péniche en feu », «Mon père apparaît près du lit, dans son manteau de feu $»^{16}$ La réalité est que la fille a brûlé les lettres de son père et c'est pourquoi ce dernier réapparaît avec le feu dans ses rêves. D'après la première interprétation lacanienne, cela manifeste l'impossible contact entre la fille et son père, ce qui ne cesse d'ailleurs de revenir dans d'autres cauchemars : « Mon père est debout sur une péniche en feu. Je dérive sur l'eau, je tente de le rejoindre. Mais les têtes coupées happent mes cheveux, me tirent en arrière ", " Mon père m'appelle au secours, me tend la main. J'essaie de l'attraper, mais nos mains ne se rejoignent pas $» \cdot{ }^{17} \mathrm{En}$ revanche, l'apparition répétitive du père associé à des images de feu exprime aussi le désir de l'écrivaine de faire revivre et d'immortaliser son père, en accord cette fois avec la deuxième interprétation lacanienne. Le feu est donc à la fois la mort et la vie. Le feu, d'après Ronald Laing, peut être aussi «l'incertain vacillement de la vitalité intérieure de l'individu, il peut être une puissance extérieure destructive qui le détruira $»{ }^{18}$ Bachelard, quant à lui, dit que le feu est un phénomène qui peut tout expliquer : "Il monte des profondeurs de la substance et s'offre comme un amour. Il redescend dans la matière et se cache, latent, contenu comme la haine et la vengeance.$^{19}$ Ainsi, les images délirantes sont toutes liées à la mort telles que : têtes coupées, chiens de l'enfer, chien à trois têtes dont la gueule crache du feu, père mort dans un manteau de feu, avec une boule de feu, sur une péniche de feu...

16 L'oiseau est également un signe-symbole qu'on rencontre à plusieurs reprises dans la trilogie. Il est un symbole de la migration (oiseau migrateur). Chez Linda Lê, il s'agit de l'oiseau affamé, l'oiseau chassé, l'oiseau blessé, l'oiseau aux ailes coupées. La Manchotenarratrice est appelée "l'oiseau de mauvaise augure Albatroce », «l'oiseau de malheur exilé des huées». D'ailleurs, le père mort qui s'envole vers le ciel dans les derniers passages des Trois Parques est appelé « l'oiseau Lear », l'oiseau qui enfin « cessa de battre des ailes [...] se laissa tomber dans le fleuve noir ${ }^{20}{ }^{20} \mathrm{Et} \mathrm{l'oiseau} \mathrm{aux} \mathrm{ailes} \mathrm{coupées,} \mathrm{blessées,}$ écrasées auquel la narratrice du deuxième volet s'identifie évoque bien l'idée de l'impossible retour. Les « ailes » font entendre fortuitement le sigle de notre écrivaine $\mathrm{L}$. L. Baudelaire a d'ailleurs utilisé l'image de l'albatros, un oiseau isolé parmi les hommes, pour définir son statut d'exil, de solitude dans le monde. Aussi le mot-valise Albatroce (albatros + atroce) est-il un autre surnom de la Manchote. On peut référer à un passage du deuxième volet où les hallucinations de la narratrice sont peuplées par les oiseaux : 
Je marche dans un parc. Les allées sont jonchées de cadavres d'oiseaux morts. À chaque pas, j'entends leurs os craquer sous mes pieds. Le charnier dégage une odeur épouvantable. Des coups de feu claquent. Je vois d'autres oiseaux battre de l'aile et tomber sur l'herbe fraîche. Les hommes de l'Organisation se sont faits chasseurs. Ils tirent sur les oiseaux par jeu. Leurs fusils sont pointés vers moi. Ils m'attendent au tournant pour m'abattre. J'avance parmi les oiseaux morts. J'entends le rire des chasseurs au loin. Et les cris des oiseaux qu'on abat. Je ramasse un oiseau blessé qui palpite encore contre mon sein. Je le caresse. Sous mes doigts, il se transforme en pierre. Une pierre tachée de sang indélébile. Je touche les oiseaux morts, ils s'envolent en nuée, puis reviennent, fondent sur moi, prêts à m'attaquer, à me picorer les yeux. Je cours en écrasant sous mes pas les ailes des oiseaux qui gisent dans l'allée. Une balle siffle à mon oreille. Une autre m'atteint dans le dos. Je m'écroule parmi les oiseaux morts. ${ }^{21}$

Le double est un thème qui hante Linda Lê. Il est certes lié au statut de l'écrivaine de double culture. Le double réside non seulement dans le dédoublement de l'auteure en personnage/narratrice mais aussi de façon très symbolique dans la perception du Moi :

Je crois qu'on n'écrit pas sans haine de soi, sans la volonté de questionner au sens où le Moyen Âge l'entend, le double qui est en soi, celui que l'on aurait voulu être, devenir, et que l'on porte comme un mort. C'est ce sentiment qui survit à l'horreur d'être né, le sentiment d'avoir perdu, d'avoir tué un frère jumeau et de n'en pas finir de régler ses comptes avec lui. ${ }^{22}$

18 C'est que son pays natal et son enfance incarnent son autre Moi perdu. Elle écrit: « La morte que je portais en moi, cette jumelle enterrée dans la maison de mon enfance et que j'avais tuée et retuée pour me donner un semblant d'existence s'était remise à vivre $»{ }^{23} \mathrm{P}$ lus d'une fois, l'écrivaine a métaphoriquement rapproché sa propre histoire de celle de deux jumeaux, la survie de l'un devant être payée par la vie de l'autre : un paysan de vingt ans qui, suite à des douleurs au ventre, se fait opérer et se voit retirer le fotus de son jumeau mort-né. Ce récit inséré dans Les Trois Parques sera repris dans Tu écriras sur le bonheur:

\footnotetext{
Ma patrie, je la porte comme ce jeune paysan portait le fœtus de son jumeau. C'est un lien monstrueux. Un lien où le pays natal, le jumeau donc, est couvé et étouffé, reconnu et dénié. Et finalement porté comme on porte un enfant mort. Ce lien monstrueux commande mon rapport à cette autre patrie, la littérature, qui naît de l'obsession d'une tare, d'une malformation, et qui s'adresse à un double. ${ }^{24}$
}

On apprend également que la Manchote ne s'est jamais remise de la séparation d'avec son frère jumeau avec qui elle avait tissé une relation incestueuse. Il s'agit à la fois d'un amour impossible et de la douleur d'être arraché à son double dont l'on ne parvient pas à faire le deuil. La question se pose dès lors à l'exilé : Que faire de son double qui est son ancien Moi ? Cette question épuise les personnages de Linda Lê dans leur quête inachevée d'une réponse insaisissable. "Chacun porte en soi un frère assassiné, il faut vivre en le ménageant ", répond l'un de ses personnages. Linda Lê, quant à elle, affirme : "En me lançant dans l'écriture et la conquête de la langue française après avoir trahi la terre et la langue de mon père $[. .$.$] je tuais mon vieux moi » .^{25}$ soit intérieur ou géographique. Les signes de rage évoqués reflètent alors une aliénation, 
un sentiment d'être étranger à soi-même et trouble dans ses relations avec le monde. En étant hanté par des images de persécution et de mort, la fille endeuillée se cherche un refuge dans les mots pour se créer d'autres je(ux) avec le feu. ${ }^{26}$

\section{La rage: autres je(ux) avec le feu}

21 L'auteure est allée jusqu'au fond de la vocifération comme un explorateur qui va «au fond de l'inconnu pour trouver du nouveau $»{ }^{27}$ La rage est ambivalente comme le feu avec lequel Linda Lê prend plaisir à jouer. Comment a-t-elle transformé la rage en extase ? Comment la rage reflète-t-elle une étape du deuil de son père? Comment s'en sert-elle pour sa création littéraire?

Aussi étonnant que cela puisse paraitre, la rage est comme une extase. C'est que l'amour servile de la narratrice pour son amant dans Lettre morte cachait son désir d'explorer l'expérience de l'humiliation jusqu'au bout. Cette prise de conscience fait que la haine et la colère qu'elle éprouvait contre elle-même se transforment en mépris de l'Autre qui est son amant: «Dans l'obstination que je mettais à assouvir mon désir de souffrance [...] je voulais voir jusqu'à quel degré d'ignominie j'allais descendre, avec quelle résignation j'allais me laisser humilier ", "Dans la solitude qui était la mienne, je ne demandais que ce qui pouvait renforcer cette solitude, contribuer à ma mise à l'écart », «Rien [...] n'aurait dû me retenir auprès de cet homme, si ce n'était mon désir de boire la coupe jusqu'à la lie, de me laisser humilier, traiter comme une esclave mendiant des restes d'amour ${ }^{28}{ }^{28}$ Ainsi, elle n'est plus l'objet qui subit la douleur mais elle est le sujet qui la chérit. C'est de cette façon qu'elle atteint la jubilation que procure la maitrise d'une situation. C'est de cette façon qu'elle détient le pouvoir de faire entrer la douleur en elle, comme à volonté.

Mais la place que prend la rage chez l'écrivaine ne se limite pas au récit-témoignage qu'elle apporte à partir de son expérience personnelle. Fortement influencée dans son inspiration par des auteurs comme Hölderlin, Kierkegaard, Artaud, elle mesure les effets proprement stylistiques et littéraires de cette expérience sur l'écriture elle-même. Quel est le rapport de la rage et de la folie à sa littérature ? Linda Lê a pleinement conscience de la fascination qu'exerce la folie sur elle, et elle confie précisément à l'écriture la mission de maîtriser cette dernière pour qu'elle ne devienne pas une fin en soi :

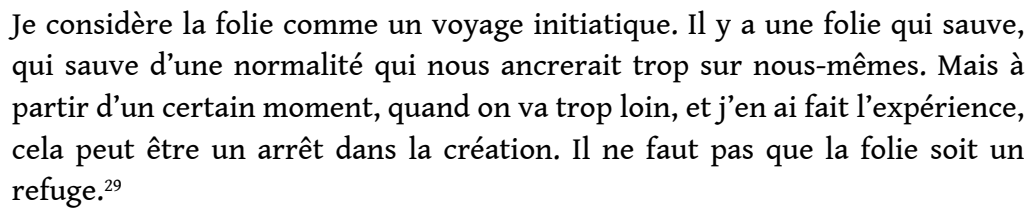

24 Au terme de cette crise qu'elle a qualifiée de très "salutaire ", la déraison lui a fait retrouver la raison. Si la rage lui a permis d'exprimer son refus de la réalité douloureuse, l'écriture à laquelle elle s'est vouée dit en revanche avec force son refus de la folie. Étant la maitresse des personnages qui errent dans la solitude et la crise et qui s'immolent dans le malheur, elle réalise que :

... ce sont des moments non seulement de désarroi... mais plus que cela : des moments de complète stérilité... Les crises empêchent non seulement d'avoir un quelconque rapport aux autres et au monde mais aussi d'avoir la capacité nécessaire pour affronter les mots. ${ }^{30}$ 
Lê sont un mélange où l'on ne peut distinguer les expériences personnelles de celles d'autrui ou de scènes imaginées. Mais c'est quand on la raconte que la vie prend un sens. Si bien que, quand Linda Lê peut faire le récit de sa crise, elle peut aussi s'en distancier. La trace écrite ne permet pas seulement de pérenniser le souvenir : elle favorise aussi la maitrise des affres. La mise en texte de l'expérience vécue a pour but de la faire entendre et de mieux la partager. Ces livres-offrandes, considérés comme " une stèle en papier » par l'auteure, lui ont permis de se réconcilier avec elle-même. 
La rage est donc une expérience pathologique, créative et thérapeutique chez Linda Lê. Elle apparaît à la fois comme expérience authentique et œuvre littéraire: ou, plus exactement, ce sont la forme et la création littéraires, avec tout le symbolisme qu'elles mettent en œuvre, qui ont permis à l'auteure de l'exprimer au mieux, et donc de la surmonter. Un remède à la rage a été trouvé dans la folie d'écrire.

\section{NOTES}

1. Entretien avec Catherine Argand, Lire, Avril 1999 [Site consulté le 20/04/2017: http:// www.lexpress.fr/culture/livre/linda-le_803102.html]

2. Emmanuel KANT, Critique de la raison pure (nouvelle traduction française par A. Tremesaygues et B. Pacaud), Paris, Félix Alcan Editeur, 1905.

3. Propos recueillis par Thierry Guichard, «Linda Lê : 'Tout doit être sacrifié pour l'écriture' », Le Matricule des anges, $\mathrm{n}^{\circ} 13,1995, \mathrm{p} .6$.

4. Entretien avec Catherine Argand, op. cit.

5. Correspondance privée avec Linda Lê (16 juin 2011).

6. Correspondance privée avec Linda Lê.

7. Linda LÊ, Les Trois Parques, Paris, Christian Bourgois, 1997, p. 36.

8. Ibid., p. 17.

9. Ibid., p. 81-82.

10. Sigmund Freud, Totem et tabou: Interprétation par la psychanalyse de la vie sociale des peuples primitifs (traduit de l'allemand par Serge Jankélévitch), Paris, Payot, 1965, p. 220.

11. Linda Lê, Voix : une crise, Paris, Christian Bourgois, 1998, p. 10, 14-15, 18.

12. Ibid., p. 28, 29.

13. Ibid., p. 48.

14. Ibid., p. 58-59.

15. Linda Lê, Lettre morte, Paris, Christian Bourgois, 1999, p. 52, 76-78, 81, 82.

16. Linda LÊ, Voix : une crise, op. cit., p. 27, 31, 37, 39, 45.

17. Ibid., p. 39, 49.

18. Ronald D. LAING, Le Moi divisé : De la santé mentale à la folie, Paris, Stock, 1993, p. 59.

19. Gaston BACHELARD, La Psychanalyse du feu [1949], Paris, Gallimard, 2006, col. «Folio essais », p.

23.

20. Linda LÊ, Les Trois Parques, op. cit., p. 245.

21. Linda LÊ, Voix : une crise, op. cit., p. 35-36.

22. Entretien avec Catherine Argand, op. cit.

23. Linda LÊ, Autres jeux avec le feu, Paris, Christian Bourgois, 2002, p. 41.

24. Linda LÊ, Tu écriras sur le bonheur, Paris, PUF, 1999, p. 330.

25. Linda LÊ, Le Complexe de Caliban, Paris, Christian Bourgois, 2005, p. 51-52.

26. D'après le titre d'un livre de Linda LÊ « Autres jeux avec le feu », paru chez Christian Bourgois en 2002.

27. Ce vers de Baudelaire a été repris par Linda Lê pour l'intitulé d'un de ses recueils d'essais.

28. Linda Lê, Lettre morte, op. cit., p. 82, 83, 90. 
29. Romaric SANGARS, Linda Lê: Discrète Antigone [Site consulté le 20 avril 2017: http:// www.chronicart.com/digital/linda-le-discrete-antigone/].

30. Propos recueillis par Thierry GUICHARD, « Franchir les frontières ", Le Matricule des anges, $\mathrm{n}^{\circ} 86$, 2007, p 20.

31. Ibid.

32. Correspondance privée avec Linda Lê.

33. Marie-France ETIENNE, "Linda Lê ou les jeux de l'errance ", Tangence, Hiver 2003, n 71, p. 79-80.

34. Entretien avec Linda Lê par Catherine Argand, op. cit.

\section{ABSTRACTS}

Cet article porte sur l'écriture à la fois recherchée et troublante d'une trilogie aux couleurs autobiographiques consacrée à la mort du père. Les troubles résident non seulement dans la matérialité des mots mais aussi dans une rupture des normes de communication (cohérence, réalisme) qui rend le texte difficile à aborder. Chez Linda Lê, les tentatives de suicide sont une révolte contre le Moi, la solitude un refus de l'Autre et l'anathème une façon de prendre Dieu pour cible. Les pulsions se déchargent dans le langage et l'écriture est une nécessité pour faire le deuil et un pharmakon contre la folie. On est entraîné dans un duel où le silence est détruit par le bruit de voix vociférantes, comme signe de déchaînement, et le bruit par le silence, comme signe d'apaisement.

This article focuses on the writing style, both studied and unsettling, of a trilogy, with autobiographical tones, devoted to the death of the father. The Trouble lies not only in the choice of words but also in a breach in the communication standards (consistency, realism) which makes the text difficult to apprehend. With Linda Lê, suicidal attempts are a rebellion against the Self, the solitude a rebuttal of the Other, and the anathema is aimed at God. The impulses are discharged in the language. Writing is necessary to go through mourning and it is a pharmakon against madness. The reader is dragged into a duel where silence is destroyed by the noise of vociferous voices, as a sign of fury, and noise destroyed by silence, as a sign of appeasement.

\section{AUTHOR}

\section{THU THUY BUI}

Titulaire d'un doctorat de Lettres modernes de l'Université Lumière Lyon 2, Thu Thuy Bui mène actuellement des recherches sur la littérature de l'exil, notamment dans une approche interculturelle. Elle porte un intérêt particulier à la relation entre littérature et psychanalyse. Elle s'intéresse également à la littérature populaire vietnamienne en rapport avec la musique. Elle traduit des textes littéraires du français au vietnamien et vice-versa.

Thu Thuy Bui obtained her Ph. D. In Modern Literature at Université Lumière Lyon 2 in 2012. Her research topics include the exile writing, in particular from an intercultural perspective, as well as the relation between literature and psychoanalysis. She is also interested in popular Vietnamese literature in relation with music. She translates books from French to Vietnamese and vice-versa. 